\title{
Percutaneous Angioplasty in Diabetic Patients with Critical Limb Ischemia and Chronic Kidney Disease
}

\author{
Laura Giurato, Roberto Gandini, Marco Meloni, Enrico Pampana, Valeria Ruotolo, \\ Valentina Izzo, Sebastiano Fabiano, Costantino Del Giudice, Luigi Uccioli \\ Department of Internal Medicine of University of Tor Vergata, Rome, Italy \\ Email: luccioli@yahoo.com
}

Received April 23, 2013; revised May 25, 2013; accepted June 25, 2013

Copyright (c) 2013 Laura Giurato et al. This is an open access article distributed under the Creative Commons Attribution License, which permits unrestricted use, distribution, and reproduction in any medium, provided the original work is properly cited.

\begin{abstract}
Introduction: Diabetes and Chronic Kidney Disease (CKD) are two strong risk factors for peripheral arterial disease (PAD) and Critical Limb Ischemia (CLI). Further renal insufficiency increases the risk of non healing wounds and major amputation. Primary amputation rates of $22 \%$ to $44 \%$ have been reported for ischaemic foot lesion in End-Stage Renal Disease (ESRD) patients. In our study we evaluated the outcomes after Percutaneus Transluminal Angioplasty (PTA) in diabetic patient in relation to different CKD classes. Materials and Methods: We studied a group of 456 diabetic patients with PAD complicated by foot lesion who underwent PTA because of a CLI. According to the estimated Glomerular Filtration Rate (eGFR $\mathrm{mL} / \mathrm{min} / 1.73 \mathrm{~m}^{2}$ ) we divided the patients into five CKD groups: group 1 eGFR $>90$, group 2 eGFR 90 - $60(n=160)$, group 3 eGFR $60-30(n=152)$, group 4 eGFR $30-15(n=34)$ and group $5<15$ or in ESRD) $(n=60)$. The following outcomes were recorded: alive without major amputation, alive with major amputation and death. The follow-up was $16.7 \pm 14.3$ months. Results: Alive without major amputation, alive with major amputation and death were respectively: for group $1(77.8 \%, 11.1 \%, 11.1 \%)$, for group 2 ( $74.4 \%, 12.5 \%, 13.1 \%)$, for group 3 (80.3\%, $11.2 \%, 8.5 \%)$, for group $4(82.3 \%, 8.8 \%, 8.8 \%)$. They were $60 \%, 18.3 \%, 21.7 \%$ for group 5 significantly different from the other CKD groups $\left(\chi^{2}=0.0175\right)$. Our analysis did not highlight any relationship between eGFR and outcomes and eGFR did not show any significant difference according to the different outcomes, and were respectively $60.2 \pm 1.3,61.8 \pm 3.4,63.8 \pm 3.5(\mathrm{P}=\mathrm{ns})$. Conclusion: The outcomes were similar for groups 1-4 and therefore, according to our data, they seemed not to be influenced by the decline of GFR. Outcomes worse significantly in group 5 , but this group included only patients with ESRD in dialysis treatment. Although the outcomes after PTA in group 5 was significantly worse than the other groups, still a $60 \%$ limb salvage rate was obtained with PTA also in these very fragile patients. PTA was much less aggressive than by-pass and PTA was the only method used to treat CLI in our patients. This could explain why we recorded similar outcomes in all groups despite the decline of GFR that, generally speaking, mirrors a worsening of the general clinical conditions. Worse outcomes were recorded only in group 5 and in this group dialysis by itself might be responsible of the different outcomes.
\end{abstract}

Keywords: Diabetes; Peripheral Arterial Disease; Chronic Kidney Disease; Angioplasty

\section{Introduction}

It is well known that diabetes and renal disease are two independent risk factors for peripheral arterial disease (PAD) [1]. Further, renal failure is an independent predictor of nonhealing of ischemic and neuroischemic foot lesions and it increases the risk of major amputation [2,3]. Some studies suggest that there is a clear temporal association between the incidence of foot ulcerations and major amputation and the worsening of renal state [4]. In patients with ischemic foot lesions and end stage renal disease (ESRD), a primary amputation rate of $22 \%$ to
44\% has been reported [5]. Patients with Chronic Kidney Disease (CKD) are difficult to study with imaging approach because iodinated contrast increases the risk of contrast-induced nephropathy $[6,7]$ and paramagnetic contrast agents (gadolinium) can not be used in patient with severe renal disease (estimated glomerular filtration rate $<30 \mathrm{ml} / \mathrm{min}$ per $1.73 \mathrm{~m}^{2}$ ) due to the high risk of nephrogenic systemic fibrosis [8-10]. These patients are difficult to treat and, despite acceptable results in terms of patiency after revascularization treatment, long-term outcomes are poor $[11,12]$. The aim of this study was to identify outcomes after percutaneus transluminal angio- 
plasty (PTA) in diabetic patients with critical limb ischemia complicated by foot lesion according to CKD classes as defined by Kidney Disease Outcomes Quality Initiative (KDOQI) and modified and endorsed by Kidney Disease Improving Global Outcomes (KDIGO).

\section{Materials and Methods}

From November 2002 to November 2007, a population of 456 diabetic subjects (292 men and 164 women) with critical limb ischemia and foot ulcer was followed at U.O. Diabetic Foot of University of Rome Tor Vergata and was analyzed to describe the long-term outcomes after percutaneous angioplasty. Each patient was assessed for age, sex, type and duration of diabetes, blood pressure, dyslipidemia, heart disease, kidney function, smoke. They underwent laboratory assessment as blood glucose, A1C, total cholesterol, low density lipoprotein, high density lipoprotein, triglycerides, serum creatinine, estimated glomerular filtration rate (eGFR). After this evaluation patients were divided into five groups in relation to CKD classes according to the Cockroft-Gault formula: class 1 with eGFR $>90 \mathrm{ml} / \mathrm{min}(\mathrm{n}=36)$, class 2 with eGFR 90 $60 \mathrm{ml} / \mathrm{min}(\mathrm{n}=160)$, class 3 with eGFR $60-30 \mathrm{ml} / \mathrm{min}$ ( $\mathrm{n}=152)$, class 4 with eGFR $30-15 \mathrm{ml} / \mathrm{min}(\mathrm{n}=34)$ and class 5 ESRD under dialysis treatment $(n=60)$. All patients have been examinated for foot lesions findings, evaluating dimension, deep, infection and Texas University classification. Each patient performed a carotid vessels analysis by color duplex US and a lower limbs evaluation (magnetic resonance angiography, computed tomography, color duplex US) before performing PTA. All patients were also routinely treated by dual antiplatelet therapy (aspirin with ticlopidine or clopidogrel) before the procedure and for one month after. The treatment protocol included: rapide and extensive debridement of foot lesion, mainly in presence of gangrene, oral or intravenous antibiotic therapy if requested by clinical signs of infection, revascularization by endovascular treatment as first strategy, strict follow-up. All of them were periodically evaluated after procedure: they performed debridement, medication, $\mathrm{TcPO}_{2}$ test, laboratory and therapy assessment. We reported outcomes at 12 months as: alive without major amputation, alive with major amputation and death. Statistical analysis was performed using SAS (release 6.12; SAS Institute, Cary, NC) for personal computers. Data are expressed as means \pm SEM. Comparisons between group characteristics were made with a $\chi^{2}$ test (frequency data) or ANOVA (continuous data).

\section{Results}

Baseline data are summarized in Table 1. The clinical characteristics of patients for each CKD class are similar, the only differences were related to age (patients in class 1 are younger), type of diabetes (in class 5 there were more type 1 diabetes), diastolic blood pressure and levels of ldl cholesterol (both parameters were lowest in class 5). Most of patients were aged $>65$ years, were male (64\%), had type 2 diabetes (93.5\%) and a mean duration

Table 1. Baseline clinical characteristic of $\mathbf{4 5 6}$ patients according to chronic kidney disease stage.

\begin{tabular}{|c|c|c|c|c|c|c|}
\hline CKD classes & Class $1 \mathrm{n}=40$ & Class $2 \mathrm{n}=164$ & Class $3 n=154$ & Class $4 n=38$ & Class $5 \mathrm{n}=60$ & $\mathrm{P}$ value (X ANOVA) \\
\hline Age (mean) & 62.9 & 66.9 & 70.4 & 71.5 & 71.9 & $<0.001$ \\
\hline Sex (\% male) & 67 & 70 & 51 & 53 & 71 & ns \\
\hline Type 2 diabetes (\%) & 91.5 & 96.9 & 94.1 & 91.2 & 86.6 & $<0.001$ \\
\hline Diabetes duration (years) & 20.9 & 19.4 & 20.4 & 20.5 & 19.8 & ns \\
\hline Blood glucose (mg/dl) & 159.8 & 146.9 & 151.1 & 145.8 & 139.8 & ns \\
\hline A1C (\%) & 7.98 & 8.08 & 7.39 & 9.77 & 7.17 & ns \\
\hline DBP (mmHg)) & 80.5 & 82 & 79.6 & 78.6 & 77 & $<0.001$ \\
\hline SBP (mmHg) & 138.7 & 135 & 134.9 & 135 & 134 & ns \\
\hline Total cholesterol (mg/dl) & 155.7 & 159.8 & 173.8 & 167.7 & 146.5 & ns \\
\hline HDL (mg/dl) & 36.1 & 38.2 & 42.6 & 43.6 & 41.4 & ns \\
\hline Triglycerides (mg/dl) & 126.5 & 135.5 & 140 & 137 & 163 & ns \\
\hline LDL (mg/dl) & 95.7 & 96.4 & 106.1 & 92.7 & 81.5 & $<0.001$ \\
\hline Ulcer dimension $>5 \mathrm{~cm}$ & 57.58 & 53.06 & 59.71 & 62.4 & 57.04 & ns \\
\hline Infection yes (\%) & 81.2 & 75.2 & 72 & 70 & 75 & ns \\
\hline TWC D3 (\%) & 85.7 & 78.9 & 78.63 & 76.19 & 77.5 & ns \\
\hline
\end{tabular}

DBP, diastolic blood pressure; SBP, systolic blood pressure; TWC, Texas wound classification. 
disease of 20 years. All the classes of CKD did not present an adequate diabetes control (A1C > 7\%) and a systolic blood pressure control (PAS > $134 \mathrm{mmHg}$ ). The majority of ulcers were infected and presented an extension to the bone (Texas Wound Classification D3). Only a small subgroup of patients was in ESRD under dialysis treatment (13.1\%). Surgical debridement was performed at first observation and repeated when necessary during the following control; antibiotic therapy, oral or intravenous, was continued driven by microbiological test until the resolution of clinical signs of infection. Our analyses reported the following outcomes for classes 1-4: alive without amputation (77.8\%, 74.4\%, 80.3\%, 82.3\%), alive with major amputation $(11.1 \%, 12.5 \%, 11.2 \%, 8.8 \%)$, dead $(11.1 \%, 13.1 \%, 8.5 \%, 8.8 \%)$ while outcomes for class 5 (ESRD) resulted respectively $60 \%, 18.3 \%, 21.7 \%$ $\left(\chi^{2}=0.0175\right)$ (Figure 1). These datas suggest that there was no relationship between eGFR and outcomes. Mean eGFR was equivalent for the three reported major outcomes respectively $60.2 \pm 1.3,61.8 \pm 3.4,63.8 \pm 3.5 \mathrm{P}=$ ns. It was clearly a significant impairing in terms of outcomes in patients under dialysis treatment.

\section{Discussion}

Several studies reported a correlation between kidney function and PAD. ESRD under dialysis is considered a real risk for lower limbs amputations, hospitalisation and both total and cardiovascular mortality [13-15]. The NHANES study 1999-2000 reported in its epidemiological analysis the prevalence of PAD in patients with GFR below 60 compared with the population with a GFR higher than $60 \mathrm{ml} / \mathrm{min} / \mathrm{m}^{2}$ respectively lower $3.7 \%$ and $24 \%$ [16]. Similar results are reported in a sub-analysis of ARIC study, where the authors analysed the risk to develop PAD comparing patients with eGFR $>90$ $\mathrm{ml} / \mathrm{min} / 1.73 \mathrm{~m}^{2}$ and $<60 \mathrm{ml} / \mathrm{min} / 1.73 \mathrm{~m}^{2}$ [17]. It is not completely clear the reason of high prevalence of PAD in patients affected by CKD. The classic cardiovascular risk factors, prevalent in CKD patients, could probably explain the higher presence of PAD, but it is only one of several hypothesis [18]. The alterations of mineral metabolism could play a role in the arterial disease and could be associated with increments in all-cause mortality, above all in dialytic patients [19]. Indeed the presence of PAD is related with higher cardiovascular and total mortality risk and there is a clear relationship between renal failure and both total and cardiovascular morbidity and mortality, according to the decrease of kidney function [20,21]. In diabetic patients with CLI complicated by a foot lesion, CKD increases the risk of non healing ulcer and major amputation [4]. Some studies suggest that outcomes are related to the level of kidney disease and the risk of major amputation increases according to the worsening of renal failure [3]. Venermo et al. showed that CKD is a formidable risk for major amputation of lower limbs after infrainguinal revascularization, and one-year amputation free-survival in CKD classes 3-5 was $41 \%$, lower than other CKD classes. Other significant risk factors were aged $>75$ years old and the presence of gangrene [22]. A retrospective review conducted by Owens C.O. analyzed the outcomes of a cohort of 456 patients who underwent by-pass surgery treatment of lower limbs because of PAD. The results showed as CKD staging identifies a curve of risk of major amputation among patient with renal disease. The 5-year survival rates by CKD class were CKD 1-2 57\%, CKD 3 46\%, CKD 4 23\% and CKD 5 9.5\%. After adjustment CKD 4 and 5 were significant predictors of mortality and major amputation $(\mathrm{P}<0.0001)$ [12]. We assessed the outcomes of our diabetic population with CKD who performed PTA because of a CLI. We evaluated our results according to the level of renal disease expressed as a different stage of CKD and we found that no differences in outcomes are observed in the different CKD classes beside the group 5 in which significant worse outcomes are recorded. Our results are significantly different from the other literature data either for the patients in dialysis and for the remaining classes of CKD. In fact for dialysis patients we found a higher limb salvage rate and a reduced major amputation and death

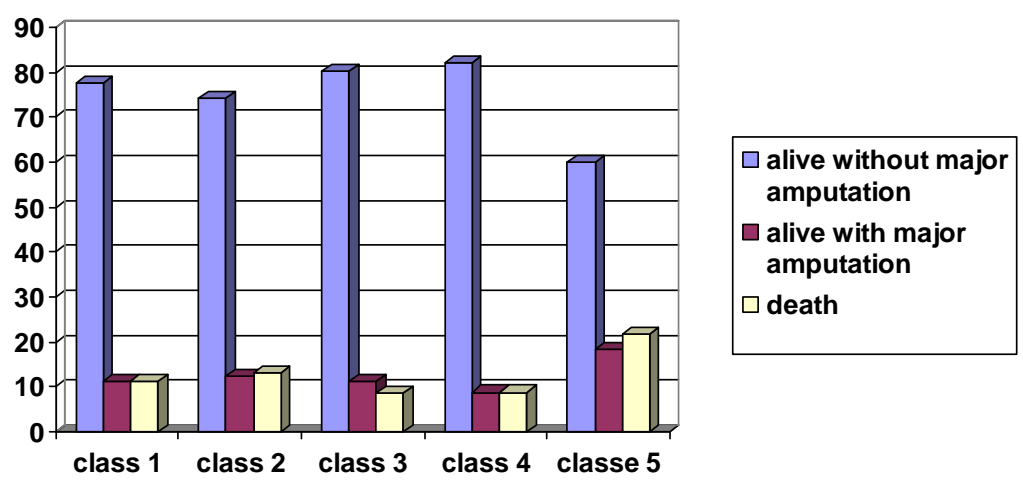

Figure 1. In the figure above the outcomes of our cohort divided for CKD classes $\left(\chi^{2}=\mathbf{0 . 0 1 7 5}\right)$. 
in an unselected population. The same positive results are reported regarding the CKD classes. While other authors found a worsening in the outcomes with the decline of the renal function, our data show a similar outcome for the different stage of CKD. The simplest explanation to justify these data is related to the management used to perform limb salvage that is surgical with the traditional by-pass in the majority of literature data while is endovascular in our cohort of patients. It must be underlined that PTA is much less invasive than traditional bypass and therefore more feasible in these fragile patients. This may explain the better results also in different CKD classes and the outcomes are extremely positive for all groups despite the worse clinical conditions. In conclusion, our data suggest that in terms of limb salvage, major amputation and death, ESRD can influence these outcomes as highlighted in several studies. The different levels of renal function up to class 4 do not affect these outcomes. In patients with diabetes and CKD it is important to identify and treat modifiable traditional and not traditional cardiovascular risk factors to avoid a worsening of renal disease represented by eGFR reduction and to improve the outcomes in terms of limb salvage, cardiovascular morbidity and mortality and total mortality.

\section{REFERENCES}

[1] L. Norgren, W. R. Hiatt, J. A. Dormandy, M. R. Nehler, K. A. Harris and F. G. Fowkes and TASC II Working Group, "Intersociety Consensus for the Management of Peripheral Arterial Disease (TASC II),” European Journal of Vascular and Endovascular Surgery, Vol. 33, Supplement 1, 2007, pp. S1-S75.

doi:10.1016/j.ejvs.2006.09.024

[2] L. Prompers, N. Schaper, J. Apelqvist, M. Edmonds, E. Jude and D. Mauricio, "Prediction of Outcome in Individuals with Diabetic Foot Ulcers: Focus on the Differences between Individuals with and without Peripheral Arterial Disease. The EURODIALE Study,” Diabetologia, Vol. 51, No. 5, 2008, pp. 747-755. doi:10.1007/s00125-008-0940-0

[3] M. A. Gershater, M. Löndahl, P. Nyberg, J. Larsson, J. Thörne and M. Eneroth, "Complexity of Factors Related to Outcome of Neuropathic and Neuroischaemic/Ischaemic Diabetic Foot Ulcers: A Cohort Study,” Diabetologia, Vol. 52, No. 3, 2009, pp. 398-407. doi:10.1007/s00125-008-1226-2

[4] F. L. Game, S. Y. Chipchase, R. Hubbard, R. P. Burden and W. J. Jeffcoate, "Temporal Association between the Incidence of Foot Ulceration and the Start of Dialysis in Diabetes Mellitus,” Nephrology Dialysis Transplantation, Vol. 21, No. 11, 2006, pp. 3207-3210. doi:10.1093/ndt/gfl427

[5] M. Albers, M. Romiti, C. A. Braganca Pereira, R. L. A. Fonseca and M. Da Silva Junior, "A Metaanalysis of Infrainguinal Arterial Reconstruction in Patients with ESRD,” European Journal of Vascular and Endovascular Sur- gery, Vol. 22, No. 4, 2001, pp. 294-300. doi:10.1053/ejvs.2001.1469

[6] F. Pomposelli, “Arterial Imaging in Patients with Lower Extremity Ischemia and Diabetes Mellitus," Journal of Vascular Surgery, Vol. 52, Supplement 3, 2010, pp. 81S91S. doi:10.1016/j.jvs.2010.06.013

[7] M. Heikkinen, M. Salmenperä, A. Lepäntalo and M. Lepäntalo, "Diabetes Care for Patients with Peripheral Arterial Disease," European Journal of Vascular and Endovascular Surgery, Vol. 33, No. 5, 2007, pp. 583-591. doi:10.1016/j.ejvs.2007.01.012

[8] M. R. Prince, H. Zhang, M. Morris, J. L. MacGregor, M. E. Grossman and J. Silberzweig, "Incidence of Nephrogenic Systemic Fibrosis at Two Largemedical Centers," Radiology, Vol. 248, No. 3, 2008, pp. 807-816. doi:10.1148/radiol.2483071863

[9] C. Rydahl, H. S. Thomsen and P. Marckmann, "High Prevalence of Nephrogenic Systemic Fibrosis in Chronic Renal Failure Patients Exposed to Gadodiamide, a Gadolinium-Containing Magnetic Resonance Contrast Agent,” Investigative Radiology, Vol. 43, No. 2, 2008, pp. 141144.

[10] R. Wertman, E. Altun, D. R. Martin, D. G. Mitchell, J. R. Leyendecker and R. B. O’Malley, "Risk of Nephrogenic Systemic Fibrosis: Evaluation of Gadolinium Chelate Contrast Agents at Four American Universities," Radiology, Vol. 248, No. 3, 2008, pp. 799-806. doi:10.1148/radiol.2483072093

[11] R. J. Hinchliffe, G. Andros, J. Apelqvist, K. Bakker, S. Friederichs and J. Lammer, "A Systematic Review of the Effectiveness of Revascularization of the Ulcerated Foot in Patients with Diabetes and Peripheral Arterial Disease," Diabetes/Metabolism Research and Reviews, Vol. 28, Supplement 1, 2012, pp. 179-217. doi:10.1002/dmrr.2249

[12] C. D. Owens, K. J. Ho, S. Kim, A. Schanzer, J. Lin and E. Matros, "Refinement of Survival Prediction in Patients Undergoing Lower Extremity Bypass Surgery: Stratification by Chronic Kidney Disease Classification,” Journal of Vascular Surgery, Vol. 45, No. 5, 2007, pp. 944-952. doi:10.1016/j.jvs.2007.01.025

[13] S. Rajagopalan, S. Dellegrottaglie, A. L. Furniss, B. W. Gillespie, S. Satayathum and N. Lameire, "Peripheral Arterial Disease in Patients with End-Stage Renal Disease: Observation from the Dialysis Outcomes and Practice Patterns Study (DOPPS),” Circulation, Vol. 114, No. 18, 2006, pp. 1914-1922. doi:10.1161/CIRCULATIONAHA.105.607390

[14] S. Fishbane, S. Youn, E. Flaster, G. Adam and J. K. Maesaka, "Ankle-Arm Blood Pressure Index as a Predictor of Mortality in Hemodialysis Patients," American Journal of Kidney Diseases, Vol. 27, No. 5, 1996, pp. 668672. doi:10.1016/S0272-6386(96)90101-8

[15] A. O’Hare and K. Johansen, “Lower-Extremity Peripheral Arterial Disease among Patients with End-Stage Renal Disease," Journal of the American Society of Nephrology, Vol. 12, No. 12, 2001, pp. 2838-2847.

[16] A. O’Hare, D. V. Glidden, C. S. Fox and C. Hsu, "High Prevalence of Peripheral Arterial Disease in Person with 
Renal Insufficiency. Results from the National Health and Nutritional Examination Survey. 1999-2000," Circulation, Vol. 109, No. 3, 2004, pp. 320-323. doi:10.1161/01.CIR.0000114519.75433.DD

[17] K. Wattanakit, A. R. Folsom, E. Selvin, J. Coresh, A. T. Hirsch and B. D. Weatherley, "Kidney Function and Risk of Peripheral Arterial Disease: Results from the Atherosclerosis Risk in Communities (ARIC) Study," Journal of the American Society of Nephrology, Vol. 18, No. 2, 2007, pp. 629-636. doi:10.1681/ASN.2005111204

[18] B. G. Jaar, L. C. Plantinga, B. C. Astor, N. E. Fink, C. Longenecker and R. P. Tracy, "Novel and Traditional Cardiovascular Risk Factors for Peripheral Arterial Disease in Incident-Dyalisis Patients," Advances in Chronic Kidney Disease, Vol. 14, No. 3, 2007, pp. 304-313. doi:10.1053/j.ackd.2007.04.005

[19] R. N. Foley, P. S. Parfrey, J. D. Harnett, G. M. Kent, L. $\mathrm{Hu}$ and R. O’Dea, "Hypocalcemia, Morbidity, and Mortality in End-Stage Renal Disease,” American Journal of
Nephrology, Vol. 16, No. 5, 1996, pp. 386-393. doi:10.1159/000169030

[20] B. F. Culleton, M. G. Larson, P. W. F. Wilson, J. C. Evans, P. S. Parfrey and D. Levy "Cardiovascular Disease and Mortality in a Community-Based Cohort with Mild Renal Insufficiency,” Kidney International, Vol. 56, No. 6, 1999, pp. 2214-2219. doi:10.1046/j.1523-1755.1999.00773.x

[21] A. S. Go, G. M. Chertow, D. Fan, C. E. McCullock and C. Y. Hsu, "Chronic Kidney Disease and the Risk of Death, Cardiovascular Events, and Hospitalization," The New England Journal of Medicine, Vol. 351, No. 13, 2004, pp. 1296-1305. doi:10.1056/NEJMoa041031

[22] M. Venermo, F. Biancari, E. Arvela, M. Korhonen, M. Söderström and K. Halmesmäki, "The Role of Chronic Kidney Disease as a Predictor of Outcome after Revascularisation of the Ulcerated Diabetic Foot," Diabetologia, Vol. 54, No. 12, 2011, pp. 2971-2977. doi:10.1007/s00125-011-2279-1 